\title{
VISIBLE LIGHT PHOTON COUNTER TIMING MEASUREMENTS
}

\author{
L. Bagby, A. Bross, M. Johnson \\ FERMULAB', P.O. Box 500,60510, IL, USA \\ D. Casey, S. Gruenendahl, H. Zhu \\ Dept. of Physics. University of Rochester, Rochester NY 14627 \\ A. Chaves, J. Costa, L. Moreira \\ CBPFICEFET - EN, Rio de Janeiro, Brazil \\ J. Thompson \\ Dept of Physics, University of Maryland. Collge Park MD 20742
}

\section{Abstract}

The timing resolution of a VLPC/preamplifier system has been measured using a pulsed nitrogen laser light source. Several fast, low-noise, transimpedance amplifiers have been tested. To determine the timing resolution degradation due to the preamplifier circuits, a high intensity light source was employed to allow studies of signals from the VLPC with and without amplification. The test apparatus is presented, and a new setup which includes a radioactive source for use with a scintillating-fiber/VLPC is proposed.

\section{INTRODUCTION}

The Visible Light Photon Counter (VLPC) is a high-gain, high quantum efficiency, solid-state photomultiplier[1] (SSPM) developed by Rockwell International Science Center[2]. This variant of the SSPM is optimized for sensitivity to visible light and is capable of single photon counting at high rates when operated at temperatures of less than 10K. These qualities make the VLPC an ideal device for scintillating fiber ( $\mathrm{SciFi}$ ) readout in particle detectors [3]. The timing resolution measurements discussed here were motivated by questions about the applicability of VLPC/SciFi systems for longitudinal position measurements of particle showers in a SciFi detector where both ends of the fibers are instrumented. This work stems from engineering studies of a proposed upgrade to the DZero experiment at Fermi National Accelerator Laboratory. The resulus reported here are for $1 \mathrm{~mm}^{2}$ pixels and correspond to an operating temperature of $6.5 \mathrm{~K}$ with an applied bias voltage of $6.5 \mathrm{~V}$; this required a liquid helium cryostat and an associated cryogenic control system. Quantum efficiency of up to $85 \%$ and gain of up to 20.000 have been observed under similar conditions when VLPCs are illumi- nated with visible light[4]. The apparatus used for timing resolution measurements is shown in Fig.1.



Fig.1 Test setup for timing resolution measurements

The light source was a disk of scintillator/wave-shifter which produced visible light when illuminated with a pulsed UV laser, this method was employed to ensure compatibility with future studies involving scintillating fibers. The laser produced pulsed $U V$ light $(\lambda=337.1 \mathrm{~nm})$ with a width of 3 as at a frequency of $10 \mathrm{~Hz}$. The scintillator/wave-shifter was commercially manufactured from polystyrene doped with $\mathrm{p}$ terphenyl and 3-hidroxyflavone (3HF) and produced visible light $(\lambda-550 \mathrm{~nm})$ with a fluorescence decay time of 7.8 $n s[5]$. This light excited the VLPCs via a liquid light guide. Neutral density optical filters were used to control the light intensity. Measurements were made with an average of 10 detected photons. This light inteasity was determined by adjusting the filter so that the one, two, three, and four photon peaks could be observed. The filters were then adjusted to yield an average of 10 photons. A set of two Avalanche Photo Diodes (APD) was used to provide the trigger reference and to allow APD to APD timing resolution measurements. The measurements were performed with a Tektronix Digital Sig-

I Work supported by th U.S. Department of Energy under contract No. DE-ACO2-76H03000 
.al Analyzer, DSA602A[6], which provided various time measurements and statistical functions with a measured inherent time resolution of 2 ps. Fig. 2 shows the main components of the system.

\section{NOISE ISSUES AND SYSTEM TIMING RESOLUTION}

In practical applications, timing resolution can be degraded by noise. Triggering a signal at approximately half amplitude had the advantage of better noise immunity. When possible common-mode noise was rejected by using differential signals. The pulsed nitrogen laser itself was a source of both conductive and radiative noise. Various techniques were employed to reduce noise to acceptable levels including shielding, extensive grounding, opto-isolation, and other decoupling methods.

Two major effects other than noise degraded the timing resolution of the system, particularly for low light intensities. The wide variation in the VLPC output due mainly to the variation in the number of photons presented to the VLPC in each event, had a negative effect on timing resolution since a constant fraction discriminator was not used to eliminate slewing effects; this will be remedied in the next round of tests. The relatively long fluorescence decay-ime clearly dominates the resolution at the light level of a few photons; a different light source must be employed to eliminate this problem. Considering the entire system. the best electronic amplifiers appeared to have little effect on the overall timing resolution.

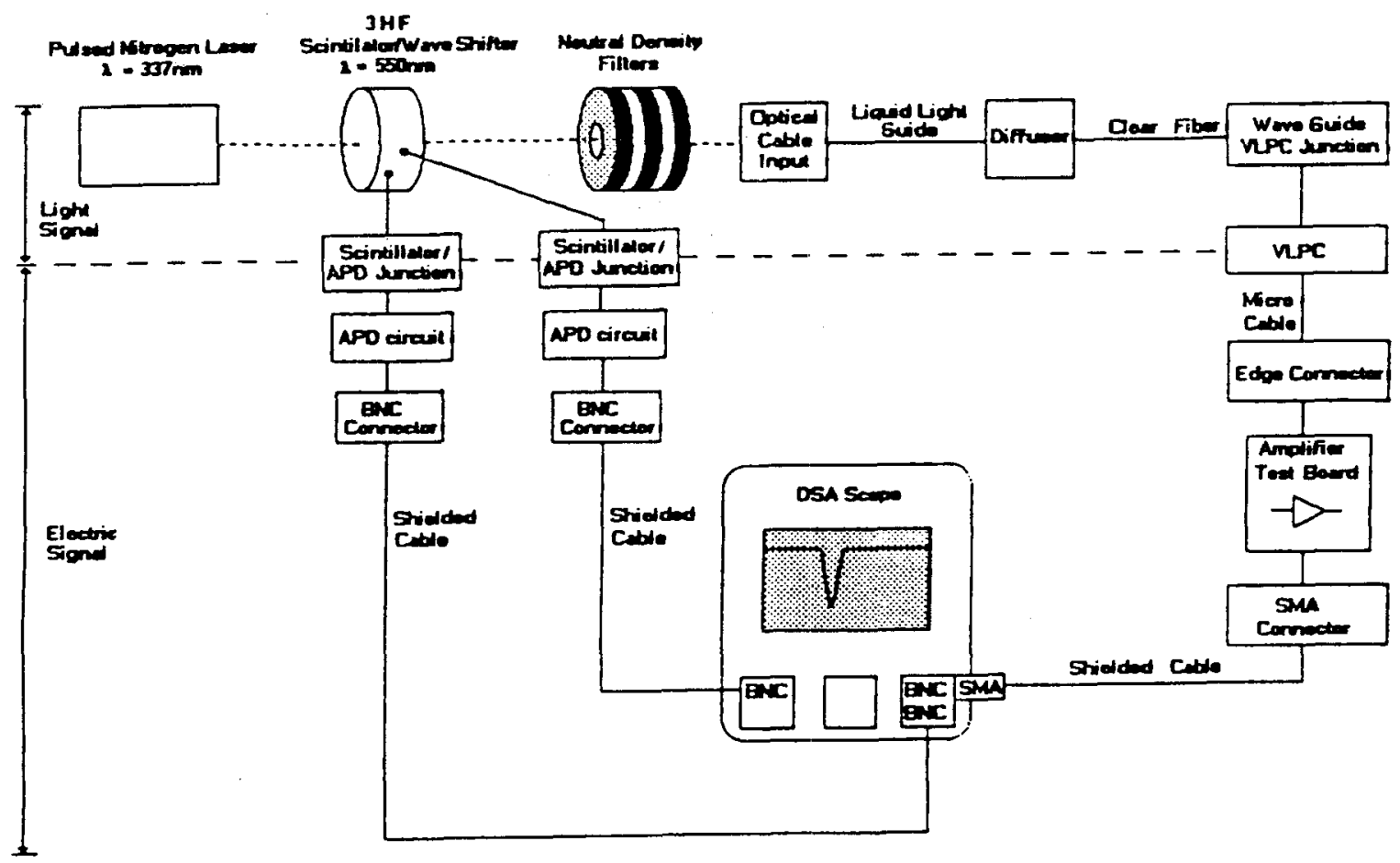

Fig. 2 System Components in the Time Resolution Measurements

\section{DEVICES}

Several devices were tested: the results presented here are from the Fermilab-QPA02, Philips-NES211, HarrisHFA1100, and a differential amplifier using discrete high frequency (HF) transistors. Timing measurements for the VLPC signal without amplification was possible only for high light intensity. The circuit used for direct VLPC readout is shown in Fig. 3.

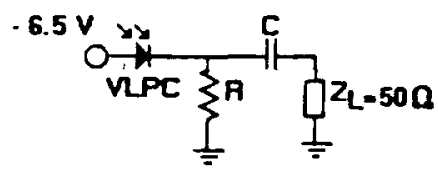

Fig.3 VLPC test circuit
A. QPAO2

The QPA02 is a high gain. 4 channel, two stage amplifier with differential output. The first stage is a transimpedance pre-amplifier that convers a charge input to a voltage output. The second stage is a differential voltage amplifier. It was developed at Fermilab using Tektronix Quickchip $2 S$ linear arrays[7][8].

\section{B. NES211}

The NE5211 is a fash, low-noise transimpedance amplifier with differential outputs developed by Philips Semiconductors[9]. It was designed primarily for fiber optic receivers. The gain is mainly determined by the first stage internal feedback resistance. 


\section{HFA 1100}

The HFAl 100 is a high-speed, wideband current feedback amplifier developed by Harris Semiconductor[10]. As its transimpedance gain decreases quickly with frequency, it has been used as a second stage amplifier with the NE5211 as a pre-amplifier. This configuration allowed high output voltage levels with good time resolution. Fig. 4 shows this test circuit. Table I depicts the main characteristics of the commercial devices tested.



Fig. 4 NE5211 + HFA 1100

Table I

Amplifiers Characteristics

\begin{tabular}{|l|c|c|c|}
\hline Device & $\begin{array}{c}\text { Input } \\
\text { Impedance }\end{array}$ & Gain & Bandwidth \\
\hline QPA02 & $200 \Omega$ & $17 \mathrm{mV} / \mathrm{fC}$ & $100 \mathrm{MHz}$ \\
\hline NE5211 & $200 \Omega$ & $\mathrm{Rr}-28 \mathrm{~K} \Omega$ & $180 \mathrm{MHz}$ \\
\hline HFA1100 & $\begin{array}{c}10 \Omega \text { (input-) } \\
50 \mathrm{~K} \Omega \text { (input+) }\end{array}$ & $\begin{array}{c}\mathrm{Rr}-500 \mathrm{~K} \Omega \\
\text { (open loop) }\end{array}$ & $850 \mathrm{Mhz}$ \\
\hline
\end{tabular}

\section{Discrete Transistor Differential Amplifier}

A differential amplifier was implemented to increase the output voltage of the NE5211 and increase the common mode noise rejection; this circuit was based on the Hitachi[11] 2SC4995, a high frequency transistor[12]. To obtain better performance during the tests, the circuit gain and bias were adjusted. Fig. 5 depicts the high-frequency transistor circuit where $\pm V$ in represent the differential outputs from the NES211 using $50 \Omega$ termination.

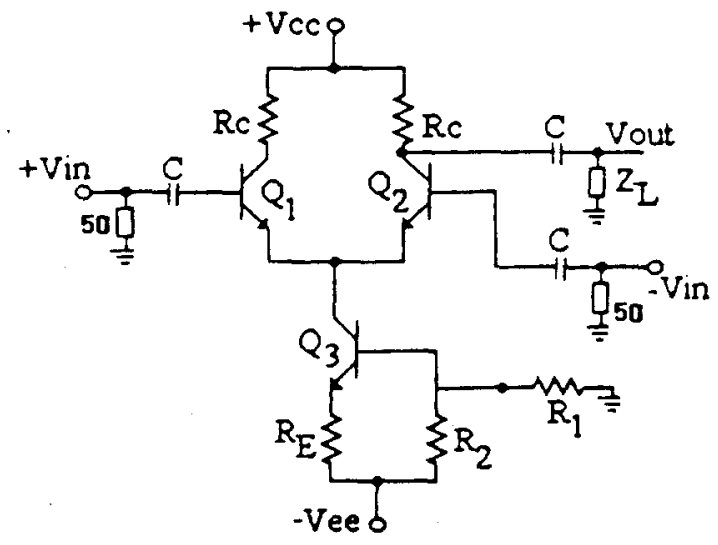

Fig. 5 Discrete HF transistor differential amplifier
Timing measurements were made using the APD as a trigger source; the measured time difference was given by the VLPC or amplifier output crossing a reference voltage chosen to be approximately the half amplitude of the signal. The reported resolution was one standard deviation of the time difference measurement distribution as determined by the DSA602A. This distribution was Gaussian to a good approximation. To reduce the effects of random noise, we required that the VLPC output crossing be found within a narrow window which covered the full leading edge of a typical pulse; crossings before or after this time were ignored by the instrument. The effects of the trigger circuit on timing resolution was studied as shown in Fig.2. Using one APD as a trigger and a second identical APD as the second time reference yielded a combined resolution of 68 ns; this indicated that the trigger conditions were a negligible contribution to the overall resolution.

Figs. 6 and 7 show the timing performance of the tested devices. All timing resolution data was measured using at least 5000 samples, in continuos mode. All output amplitudes were measured in average mode. Fig. 8 shows the VLPC output signal for $\mathrm{R}-100 \mathrm{~K}$.

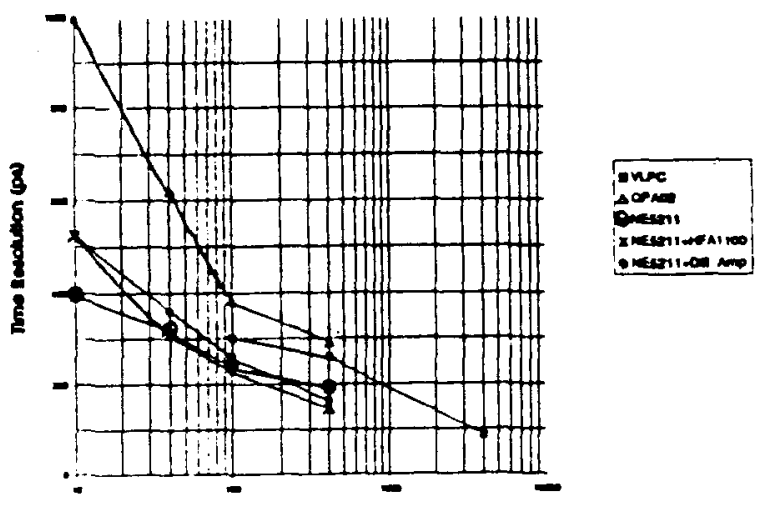

Lot then enderen

Fig. 6 Time Resolution vs. Light Intensity



Fig. 7 Output Amplitude vs. Light Intensity 


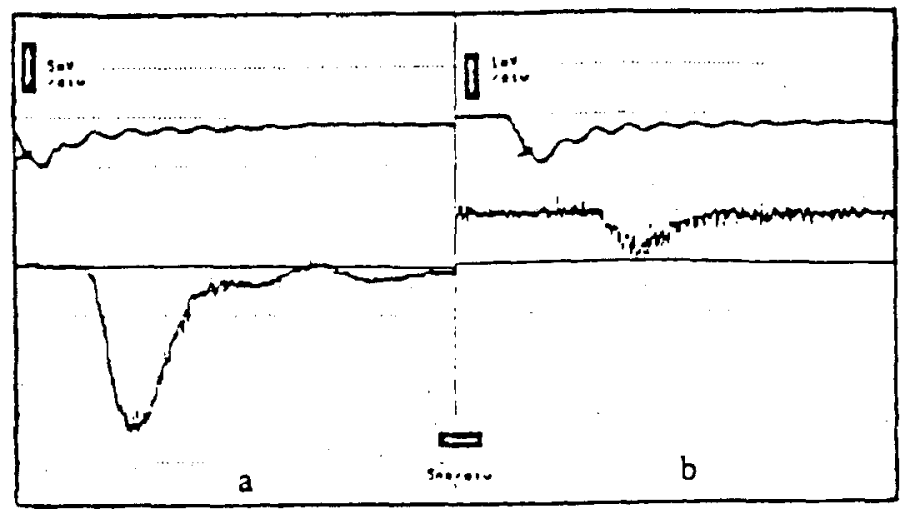

Fig. 8 VLPC signal for $R=100 \mathrm{~K}$ : a) 4000 photons; b) 100 photons

The NES211 amplifier, as a single device, had better timing resolution than the others but had relatively low gain. For a light intensity of about 10 detected photons, the output voltage signal was less than the threshold level of many commercial discriminators. The QPA02 had much higher gain but its resolution was not competitive. The NES211 used as a front-end amplifier with a differential amplifier as a second stage gave the best result. Two options were tested for this application: the HFA 1100 because of its high bandwidth. and the differential amplifier using the HF transistors. The first option produced better results; overall gain was high and the timing resolution was still much better than the QPA02. The second option produced results close to the first in terms of timing resolution. The results from this amplifier are preliminary and optimization is still in progress. The new Philips NE5223 has a higher transimpedance gain (RT-100K) and low input noise. It should improve the frontend performance.

The best electronic amplifiers had little effect on the overall timing resolution of the system as suggested in studies of the raw VLPC output signal using only the internal amplifier of the DSA602A. Fig. 9 shows the timing resolution of the VLPC output as a function of light intensity.



Fig. 9 Timing Resolution vs. Light Intensity for VLPC output
The logarithmic functional form is evident in the figure. Extrapolating the fit to a detected light intensity of 10 photons yields a predicted resolution of 450 ps without the influence of additional amplification.

\section{FURTHER DEVELOPMENTS}

These results are from preliminary studies to determine the efficacy of using timing measurements of scintillating fibers instrumented with VLPCs to determine the position along the fiber from which detected photons originated. With a refractive index of approximately 1.6 in a fiber, a 500 ps time resolution corresponds to a position resolution of $10 \mathrm{~cm}$. This resolution is acceptable for many applications; and with about ten detected photons produced by minimum ionizing paricles in a scintillating fiber, such performance seems possible based on these measurements. This goal together with experience gained in the tests reported here suggests new measurements that will clarify these results.

There are two different timing measurements that are of interest: the inherent resolution of the VLPC as a function of light intensity, and the VLPC/SciFi system as a whole for particle detection.

The inherent timing resolution of the VLPC device was not addressed for low light intensities. Aside from complications arising from light and signal dispersion, both the puise length of the laser and the decay time of the wave-shifter adversely affected the measured resolution due to the photon statistics. This will be addressed by utilizing a faster visible light laser diode and injecting the photons directly into the light guide: this may also have the benefit of reducing the shock noise associated with the spark-gap trigger in the current laser. Slewing effects will be reduced with the use of a constant fraction discriminator. For the highest light intensities discussed, these effects are not an issue suggesting that the results reported here are applicable.

The timing resolution of a working Scifi system can be most easily studied by using a collimated radioaceive source to irradiate a scintillating fiber instrumented at both ends. In this case the two ends of the fiber are sources of both the trigger and the stop-time reference; the distribution of time differences can be used to infer the resolution of a single VLPC assuming that the two devices have identical timing characteristics. A laser with a separate trigger pickoff can be used to illuminate the fiber for comparison with results obtained with the source.

Aside from better measurement techniques, developmeats of new amplifiers and VLPCs suggest even better system performance. Most promising among the aroplifiers is the new Philips-NE5223 device which has better reported performance (gain, bandwidth and noise) than the PhilipsNE5211 which was considered here. The development of a new version of the VLPC with a factor of five higher gain also points toward potentially better resolution for low light intensities. Tests are planned for each of these improvements. 


\section{REFERENCES}

[1] M.D. Petroff, M.G. Supelbrook, and W.A. Kleinhans, Appl. Phys. Lell. SI(6), 1987, pp 406-408.

(2) Rockwell Intemational Science Center, Anaheim. Califomia.

[3] F. Borcherding and M. Johnson - Fermilab, J. Cosia and L. Moreira - Lafex/Cefet - EN, Front End Signal Processing for the DZero VLPC System, Fermilab 1993.

[4] M. Atac et.al., "Scintillating Fiber Tracking at the SSC Using Visible Light Photon Counters, 'Fifth Intemational Pisa Con. ference on Advanced Detectors, May 1991.

[5] R.Ruchti et.al.. "Performance of Multiclad Scintillating and Waveguide Optical Fibers Read Out with Visible Light Photon Counters. "SPIE Intemational Symposium on Optics Inaging and Instrumentation, july 1993.

[6] Tekuronix, Wilsonville, Oregon.

[7] R.J. Yarema, ASIC Design at Fermilab, Conference on Electronics for Future Colliders, Chestnut Ridge, NY, May 1991.

[8] T. Zimmerman, A High Speed, Low noise ASIC Preamplifier for Silicon Surip Detectors, Fermilab.

[9] Philips Semiconductors, Sunnyvale, Califomia.

[10] Harris Semiconductor, Melboume, Califomia.

[1] Hitachi, Brisbane, Califomia.

[12] Alan Rudge, Comparison of Charge Collection in Semiconductor Detectors and Timing Resolution. Using a Sub-nanosecond transimpedance Amplifier, CERN Switzerland. 